rev.relac.int.estrateg.segur.13(2):123-151,2018

\title{
La Organización del Tratado del Atlántico Norte y la ampliación al Este: ihasta Ucrania?**
}

\author{
María Martínez Carmena**
}

\section{Resumen}

En este estudio se analiza la política de ampliación de la OTAN durante la Posguerra Fría, y las razones del actual estancamiento de la denominada política de puertas abiertas. Si bien es probable que todos los Estados balcánicos acaben integrados en la OTAN, otros Estados han sido configurados por parte de Rusia como el muro infranqueable de este proceso ampliador. En este sentido, Georgia y, de forma clara, Ucrania han marcado un punto de inflexión en la relación entre la OTAN y Rusia. El "factor Rusia" siempre ha determinado el avance de la Alianza Atlántica hacia el Este de Europa, pero actualmente los miembros de la OTAN, principalmente los socios europeos más relevantes, podrían verse afectados por graves implicaciones de orden político,

* Artículo en el marco del proyecto nacional "Los muros y el derecho internacional contemporáneo: implicaciones para la seguridad, la dignidad de la persona y el desarrollo sostenible" (DER201565486-R), financiado por el Ministerio de Educación, Cultura y Deporte.

** Doctora en Derecho de la Universidad de Castilla-La Mancha, España. Profesora de Derecho Internacional Público y Relaciones Internacionales en la Facultad de Ciencias Jurídicas y Sociales de Toledo, Universidad de Castilla-La Mancha. Correo electrónico: maria.martinez@uclm.es. 
económico y geoestratégico (energía, coordinación para combatir el terrorismo yihadista, etc.) si su relación con Rusia empeora. Por ello, se considera adecuado que la estrategia de la ampliación en esta etapa atienda a todas las variables, revise sus objetivos y no se realice de forma precipitada, dado que la reconstrucción de la relación con Rusia es fundamental para que exista un equilibrio euroasiático.

Palabras clave: Ampliación de la OTAN; Posguerra Fría; Rusia; Ucrania.

\title{
NATO and its Enlargement to the East: To Ukraine?
}

\begin{abstract}
This study analyzes NATO's enlargement policy during the post-Cold War and the reasons for the current stagnation of the so-called "open door policy". While it is likely that all Balkan states end up incorporated into NATO, other states have been regarded by Russia as the insurmountable wall of this enlargement process. In this sense, Georgia and, clearly Ukraine, have marked a turning point in the NATO-Russia relationship. The "Russian factor" has always determined the advance of the North Atlantic Alliance towards Eastern Europe, but currently NATO members, especially the most relevant European partners, could experience serious political, economic and geostrategic effects (energy, coordination to combat jihadist terrorism) if its relationship with Russia worsens. Thus, it is suggested that the expansion strategy at this stage covers all the variables, reviews its objectives and is not pursued hastily because reconstructing the relationship with Russia is fundamental to the existence of a Euro-Asian balance.
\end{abstract}

Keywords: NATO enlargement; post-Cold War; Russia; Ukraine.

\section{A Organização do Tratado do Atlântico Norte e a ampliação ao Leste: até a Ucrânia?}

\section{Resumo}

Neste estudo analisa-se a política de ampliação da OTAN durante a Pós-guerra Fria, e as razões do atual estancamento da denominada política de portas abertas. Se bem que é provável que todos os Estados balcânicos acabem integrados na OTAN, outros Estados têm sido configurados por parte de Rússia como o muro infranqueável deste processo ampliador. Neste sentido, Geórgia e, de forma clara, a Ucrânia têm marcado um ponto de inflexão na relação entre a OTAN e a Rússia. O "fator Rússia" sempre tem determinado o avanço da Aliança Atlântica ao Leste da 
Europa, mas atualmente os membros da OTAN, principalmente os sócios europeus mais relevantes, poderiam ver-se afetados por graves implicações de ordem política, econômica e geoestratégica (energia, coordenação para combater o terrorismo jihadista, etc.) se sua relação com a Rússia piora. Por isso, se considera adequado que a estratégia da ampliação nesta etapa atenda a todas as variáveis, revise seus objetivos e não se realize de forma precipitada, já que a reconstrução da relação com a Rússia é fundamental para que exista um equilíbrio euroasiático.

Palavras-chave: Ampliação da OTAN; Pós-guerra Fria; Rússia; Ucrânia.

\section{La decisión de ampliar la Organización del Tratado del Atlántico Norte al centro y este de Europa: una cuestión de supervivencia}

El final de la Guerra Fría conllevó una profunda crisis existencial dentro de la Alianza Atlántica, que desembocó en una renovación funcional y de composición de esta. Tras la caída del Muro de Berlín, la Organización del Tratado del Atlántico Norte (OTAN) fue consciente de que sobrevivir en el nuevo escenario estratégico surgido del "deshielo" requería una profunda revisión de sus funciones de seguridad. Unido a esta diversidad funcional en la nueva etapa, la Alianza Atlántica también debía considerar los nuevos Estados que podían formar parte de la organización. El debate político sobre la conveniencia de la ampliación se encontraba, en realidad, íntimamente ligado a la configuración de una nueva OTAN, y resultó ser uno de los pasos más trascendentes de la organización. La caída del Telón de Acero, la desaparición del Pacto de Varsovia y el acceso a la independencia política de numerosos Estados de Europa Central y Oriental habían modificado radicalmente el mapa de la Europa dividida de antaño y, por tanto, la visión geoestratégica de la OTAN de cara al futuro.

Los antiguos Estados satélite pronto mostraron su deseo de formar parte de la OTAN. En líneas generales, su objetivo prioritario en la OTAN radicaba en obtener la garantía de defensa territorial e independencia políticas dentro de sus respectivas fronteras, gracias al paraguas defensivo proporcionado por el artículo 5 del Tratado Atlántico Norte (TAN). El anhelo de los antiguos Estados de vincularse a la OTAN manifestaba en igual medida su afán por desvincularse de Rusia y de la etapa histórica anterior, y así aminorar la influencia de esta potencia en su futuro.

La Federación Rusa, muy debilitada al inicio de la Posguerra Fría, actualmente conserva su papel como potencia económica y política mundial, un rol que está siendo especialmente enfatizado por la política exterior del presidente 
Vladimir Putin. No obstante, se trata de un gigante sobre el que Occidente mantiene numerosas reticencias e interrogantes (Ferguson, 2010, p. 20). Y no es para menos. Desde 2008, tras la contienda en Georgia y, posteriormente, con la anexión de la península de Crimea en 2014, Rusia ha demostrado que está dispuesta, si lo considera preciso, a defender sus intereses contraviniendo la legalidad internacional.

Rusia nunca ha escondido su disconformidad con el proceso ampliador de la Alianza Atlántica y otras decisiones que afectaban a sus fronteras; antes bien, ha manifestado públicamente su desacuerdo con continuas advertencias a la OTAN. Ya en 1999 amenazó a Alemania sobre el posible desequilibrio internacional que podría causar la futura incorporación a la OTAN de las Repúblicas Bálticas, cuyo deseo de pertenecer a la organización era manifiesto. Tampoco es un asunto menor el rechazo ruso al proyecto del sistema de defensa antimisiles de la OTAN, un problema que aún persiste en las relaciones entre la OTAN, Estados Unidos y Rusia (Müller, 2000). Por ello, y pese a que la OTAN no lo admita abiertamente como un condicionante de la ampliación, el factor de la relación con Rusia siempre ha constituido un elemento clave a la hora de admitir a nuevos Estados miembros. Un buen ejemplo de ello son las candidaturas relegadas de Georgia y Ucrania. Ambos Estados parecen ser, por el momento, un límite infranqueable para el proceso ampliador (Requena del Río, 2014, p. 13).
La política de puertas abiertas al inicio de la Posguerra Fría contó con el impulso desde Estados Unidos del presidente Bill Clinton, su principal promotor. Este dato es clave para entender que el coste de la ampliación iba a recaer principalmente en Estados Unidos (Smith, 2000, p. 131). En la Europa de la época, el canciller alemán Helmut Köhl y la "Dama de Hierro" británica, Margaret Thatcher, también fueron destacados valedores; no así Francia y España, que en reuniones informales se mostraron muy críticos con la ampliación al Este de la Alianza Atlántica (Smith, 2000, p. 131; Caracuel Raya, 2004, p. 310). Con esta división de opiniones en el contexto europeo, la ampliación venía a demostrar la delicada tensión existente entre el deseo de seguridad de los socios europeos y el rechazo al control estratégico de Estados Unidos.

En 1993, la OTAN estuvo inmersa en una profunda discusión interna para detectar elementos a favor y en contra del proceso ampliador en la Posguerra Fría, cuyo resultado fue plasmado en el conocido informe titulado "Study on NATO Enlargement" (OTAN, 1995). La Alianza Atlántica consideró como aspectos negativos del proceso ampliador, básicamente, los siguientes elementos: en primer lugar, la ampliación implicaría una nueva línea de fractura Este/Oeste; en segundo lugar, la constante susceptibilidad de Rusia; en tercer lugar, añadir más Estados conllevaría también una mayor dificultad para adoptar resoluciones 
dentro del Consejo del Atlántico Norte (CAN) u órgano supremo de toma de decisiones por consenso en la OTAN, al ser más heterogéneo y elevado el número de Estados miembros. A ello se unía, en cuarto lugar, la inestabilidad interna de algunos Estados candidatos. Finalmente, en quinto lugar, la funcionalidad y eficacia de la Alianza podían verse en entredicho, dadas las dificultades de aplicación efectiva de la cláusula de defensa mutua - consignada en el artículo 5 del TAN- a los nuevos Estados miembros (Caracuel Raya, 2004, p. 311).

Por lo que se refiere a factores positivos de la ampliación, en el citado informe se destacó especialmente la proyección de estabilidad que la OTAN podía otorgar a los nuevos Estados democráticos (cap. 2 del informe). Se entendía que la inclusión de estos en la organización sería beneficiosa a la hora de resolver y controlar mejor sus conflictos internos, precisamente porque la OTAN exige previamente a sus candidatos alcanzar unos requisitos políticos y militares mínimos. En cuanto a la siempre delicada relación con Rusia, y pese a que había sido considerada entre los aspectos negativos, el informe ventiló el asunto de forma simplista. Se estimaba que Rusia no representaba un problema real para la OTAN, al no ser un Estado miembro de esta y carecer de poder para bloquear las negociaciones (Caracuel Raya, 2004, p. 310). Obviamente, se trata de una visión poco ajustada a la realidad internacional, dado que la relación y el entendimiento de la OTAN con Rusia siempre se han perfilado como un elemento crucial, tanto para Europa como para Estados Unidos. Es decir, de facto la OTAN no se consiente una autonomía absoluta de decisión en términos políticos, ya que el "factor Rusia" necesariamente se analiza y se toma en consideración en cada ronda ampliadora.

\section{La puesta en práctica de la denominada política de puertas abiertas}

La Alianza se refiere por primera vez a su disposición para acoger nuevos Estados durante la Cumbre de Bruselas, celebrada en enero de 1994. Si bien el informe sobre la ampliación de 1995 (vide supra) fue posterior a esta Cumbre, se puede asegurar que, en realidad, tras ella, la OTAN inauguró definitivamente la política de puertas abiertas y cesó de discutir la conveniencia de la ampliación (FrançoisPoncet et ál., 2006-2007, p. 7). De hecho, únicamente quedaban por determinar las pautas del proceso, pero no así la decisión de iniciarlo. Con respecto a las pautas, la OTAN debía acordar criterios como la velocidad de la ampliación, la integración total o parcial de los nuevos aliados, la admisión de un gran número de miembros o únicamente de los Estados acondicionados a las exigencias aliadas, así como los posibles candidatos. De forma no oficial, a la cabeza de la lista estaban Polonia, Hungría y República Checa, que presionaron con mucha fuerza para lograr su ingreso, ya que consideraban que adolecían de un va- 
cío de seguridad que históricamente les había perjudicado. Por este motivo, reclamaban a los Estados occidentales que no les abandonasen de nuevo a su suerte (Law, 1999, p. 39).

Públicamente, la OTAN mantiene que los objetivos de la ampliación se dirigen fundamentalmente a aprovechar la oportunidad histórica de borrar las líneas de fractura en Europa, y así mejorar la seguridad y estabilidad de toda el "área euroatlántica". Tomando esta premisa como base, la OTAN se dispuso a considerar el deseo de pertenecer a la organización de cualquier Estado europeo que estuviese en condiciones de cumplir con los principios sobre los que la organización se asienta, así como de contribuir a la paz y seguridad del área euroatlántica.

La OTAN, de hecho, subrayó especialmente que el diseño de la ampliación respondía a la idea de evitar posibles conflictos en Europa causados por convulsiones políticas y sociales de Estados con estructuras democráticas débiles. Por tanto, la ampliación tras la Guerra Fría se enfocó políticamente como un medio óptimo para reportar estabilidad política y seguridad a los Estados de Europa Central y Oriental recién llegados a la independencia. El criterio estabilizador de la ampliación era relevante para evitar desastres como la Guerra de los Balcanes.

Con este loable deseo de estabilización como eje, la OTAN finalmente hará primar la geopolítica sobre otros criterios tenidos en cuenta respecto a los países candidatos, como la aportación defensiva de los nuevos Estados a la organización. De hecho, la contribución militar de los candidatos que fueron ingresando en la Alianza Atlántica tras la Guerra Fría puede considerarse realmente discreta, pues modificó en poca medida el potencial militar de la organización (François-Poncet et ál., 2006-2007, p. 8). Así, pues, el beneficio de seguridad que dichos Estados aportaban no podía considerarse, a priori, como un factor de peso. Siguiendo esta línea, el proceso ampliador se fue volviendo más laxo con los Estados candidatos, de modo que las rondas ampliadoras (vide infra) no dejaban lugar a que operase un verdadero cambio político y económico en los Estados aspirantes a ser miembros.

Un claro ejemplo de las debilidades o dificultades en seguridad y defensa que arrastraban los nuevos Estados miembros quedó patente en el posconflicto iraquí, tras la invasión de Irak en 2003. La falta de experiencia de Polonia para hacerse cargo de sus funciones dentro de la división multinacional que operaba entre Bagdad y Basora derivó finalmente en una abierta petición de ayuda por parte de Polonia a la OTAN, a fin de poder cumplir con sus compromisos en el terreno (Rynning, 2005, p. 130).

\section{Las rondas ampliadoras y el factor de la relación con Rusia}

A continuación, vamos a pasear, someramente, por las distintas rondas ampliadoras. En la Cumbre de Madrid de 
1997, la OTAN invitó a formar parte de la organización únicamente a tres Estados centroeuropeos: Polonia, Hungría y República Checa. La invitación oficial se escenificó en la Cumbre de Washington de 1999, con motivo de la celebración del aniversario cincuenta de la Alianza Atlántica (protocolo de adhesión del 17 de diciembre de 1997). Durante la Cumbre de Washington se emitió un comunicado que alentaba a los Estados aspirantes a esforzarse para alcanzar las condiciones exigidas por la Alianza Atlántica. En cambio, a las puertas de la OTAN se quedaron ocho Estados miembros de la Asociación para la Paz (OTAN, 7 de junio de 2017): Rumania, Eslovenia, Eslovaquia, Letonia, Lituania, Estonia, Bulgaria y la Antigua República Yugoslava de Macedonia, los cuales, a principios de 1997, también habían solicitado pasar a ser Estados miembros.

Con la vista puesta en las futuras ampliaciones, en dicha cumbre se concretó la aprobación de un Plan de Acción para Miembros (Membership Action Plan [MAP]) (OTAN, 12 de junio de 2017). El objetivo de la OTAN con este plan es proporcionar ayuda a los Estados aspirantes en su preparación para cumplir las condiciones mínimas que la Alianza exige. La OTAN se convierte así en supervisora, coordinadora, asesora y receptora de información sobre los avances de los países aspirantes. Básicamente, las condiciones requeridas se resumen en el hecho de ser Estados europeos, con capacidad para cumplir los principios del Tratado del Atlántico Norte (Estados de de- recho) y de aportar seguridad al área euroatlántica.

Tras la primera ronda ampliadora de la Posguerra Fría, en el seno de la OTAN se debatió ampliamente si la siguiente ronda debía ser menos exigente con el cumplimiento de requisitos de los países candidatos. La intención era comprobar la viabilidad de una ampliación mayor (opción big bang), dado el éxito de la llevada a cabo en la cumbre de Washington (Caracuel Raya, 2004, p. 317). Esta idea fue apoyada por los Estados candidatos en la denominada Declaración de Vilna, del 19 de mayo de 2000. La administración Bush también se inclinaba por esta opción, con vistas a conseguir una mayor estabilidad en el sureste europeo. Así, conforme a esta directriz, se eligieron siete nuevos candidatos para la siguiente ronda ampliadora: Letonia, Lituania, Estonia, Eslovenia, Eslovaquia, Bulgaria y Rumania.

La Cumbre de Praga de noviembre de 2002 estaba, en principio, destinada a "lucir" la mayor ampliación de la historia de la Alianza Atlántica. Sin embargo, la ampliación quedó empañada por los atentados terroristas del 11 de septiembre de 2001 (11-S), de modo que la lucha contra el terrorismo internacional centró la atención de dicha cumbre. El 11-S también sirvió para afianzar la relación de confianza con Rusia, pues la ampliación podía presentarse como una oportunidad de unidad defensiva frente al terrorismo (Wolff, 16 de septiembre de 2015, p. 1108). Finalmente, los candidatos se 
incorporaron a la OTAN el 26 de marzo de 2003, mediante los respectivos protocolos de adhesión.

Desde la cumbre de Praga (2002) hasta la de Bucarest (2008), la OTAN estuvo inmersa en la lucha contra el terrorismo, especialmente en Afganistán, donde había desplegado la Fuerza Internacional de Asistencia para la Seguridad (International Security Assistance Force [ISAF]), y así postergó la cuestión de la ampliación en las posteriores cumbres de Jefes de Estado y de Gobierno que la OTAN celebró (Estambul en junio de 2004, Bruselas en febrero de 2005 y Riga en noviembre de 2006).

La siguiente ronda ampliadora —la sexta en la historia de la organizacióntuvo lugar con motivo de la Cumbre de Bucarest, en abril de 2008. Los países invitados a adquirir la condición de miembros fueron los denominados Estados del Adriático: Croacia, Albania y Macedonia, los cuales ya pertenecían al Consejo de Asociación Euroatlántico (Euroatlantic Partnership Council [EAPC]), que es el sucesor del antiguo Consejo de Cooperación del Atlántico Norte, instituido este en 1997 con el propósito de aumentar la cohesión y reforzar los vínculos en beneficio de la seguridad común. Los tres Estados también trabajaban en el MAP, pero aún debían superar ciertos problemas internos antes de su incorporación a la OTAN. Preocupaban especialmente Albania y Macedonia, pues en ambos países la corrupción y el crimen organizado representaban dos graves problemas internos (François-Poncet et ál., 2006-2007, p. 10). Finalmente, solo Albania y Croacia, desde el 1 de abril de 2009, pasaron oficialmente a ser países miembros de la OTAN.

Macedonia es uno de los Estados candidatos que, a la postre, mayor debate interno generaría. Su fragilidad democrática era evidente. Entre otros asuntos, el mayor problema que lastraba a Macedonia radicaba en la falta de protección de los derechos de la minoría albanesa. La OTAN ha alabado el esfuerzo que en estos años hizo el país balcánico para construir una sociedad multiétnica. De hecho, en agosto de 2001 tuvo lugar una intervención de la OTAN en territorio macedonio "a solicitud" del entonces presidente Boris Trajkovski. Se trató de la operación Essential Harvest, cuyo objetivo era incautar armas de las milicias albanesas en territorio macedonio y destruirlas. De este modo, quedaría asegurado el respeto al alto el fuego, pues la pequeña república se encontraba a punto de caer en una guerra civil entre extremistas albaneses y macedonios. La intervención se "prorrogó" con la operación Amber Fox, bendecida por la Resolución 1371 del Consejo de Seguridad; esta vez, tuvo el fin de proteger a los supervisores de la Unión Europea (UE) y de la Organización para la Seguridad y la Cooperación en Europa (OSCE) en su misión en dicho país (OSCE-EU Monitoring Mission). Una vez acabada esta tarea, el gobierno macedonio solicitó la presencia de la OTAN en una nueva misión (Allied Harmony), a fin de ejercer labores de 
coordinación, supervisión y asistencia a este gobierno en cuestiones militares, siendo traspasada esta responsabilidad a la UE en 2003.

Finalmente, este no fue el principal escollo que Macedonia debía sortear. La admisión de Macedonia en la OTAN ha sido impedida por una cuestión nominativa, que aparentemente puede parecer baladí. En la Cumbre de Bucarest, y como precedente único hasta el día de hoy, se decidió condicionar la entrada de Macedonia en la OTAN a la resolución de la controversia que mantiene con Grecia y que tiene por objeto el nombre de esta pequeña república balcánica (OTAN, 3 de abril de 2008, punto 20). El veto griego durante la cumbre impidió alcanzar el consenso necesario para que Macedonia ingresase en la OTAN. El problema continúa irresuelto y la República de Macedonia (nombre que sí reconoce Turquía como válido) sigue siendo denominada Antigua República Yugoslava de Macedonia (ARYM) en los documentos de la OTAN. Este último es el nombre que Grecia admite y que viene utilizando desde su independencia de Yugoslavia en 1991.

Como es sabido, el Reino de Macedonia fue el lugar que vio nacer al rey Alejandro Magno, quien desde aquel territorio griego extendió su imperio prácticamente a todo el mundo conocido de la época. Para Grecia, constituye parte de su preciado legado histórico; en ese sentido, trata de mantener tanto la identidad cultural de la Macedonia griega como la soberanía sobre esta región septentrional en sí (con la conocida ciudad de Tesalónica como capital). Con población eslava y albanesa, la República de Macedonia no se corresponde con la Macedonia clásica incluida en la actual Grecia. En la Macedonia balcánica no nacieron Filipo II ni su hijo Alejandro Magno, el conquistador del imperio persa. La prevención de Grecia y su disconformidad a la hora de admitir como oficial el nombre de República de Macedonia se debe al temor de que este genere confusión entre el territorio griego y el balcánico, y eventualmente pueda ser objeto de reclamación por parte del pequeño Estado limítrofe con Grecia. No en vano, en 2006, la ARYM pretendió otorgar a su principal aeropuerto el nombre de Alejandro Magno, ante la indignación griega.

El 5 de diciembre de 2011, el Tribunal Internacional de Justicia de la Haya dictó sentencia favorable a la pretensión macedonia, que había alegado que el veto griego a la admisión de Macedonia en la OTAN durante la Cumbre de Bucarest violaba el Acuerdo Interino firmado por ambos Estados (1995), según el cual Grecia no impediría la entrada de Macedonia en organizaciones internacionales, y que tenía las vistas puestas en la futura adhesión macedonia a la UE y la OTAN (International Court of Justice [ICJ], 5 de diciembre de 2011).

A pesar de que aún no se haya podido admitir como Estado miembro, la ARYM ha colaborado con la OTAN activamente en las misiones de Afganistán 
y Kosovo, y sigue profundizando en reformas políticas. La puerta de la OTAN, si resuelve el carácter contencioso del nombre, sigue abierta para la república balcánica, sin que exista una traba política mayor en la organización que la oposición griega (OTAN, 2 de diciembre de 2015, puntos 5-7; OTAN, enero de 2016).

\section{Posibles nuevas ampliaciones de la Organización del Tratado del Atlántico Norte}

Actualmente, las posibles líneas de ampliación de la OTAN tienen dos direcciones bastante definidas: Balcanes occidentales, por un lado, y Cáucaso y países de la Europa Oriental fronterizos con Rusia, por otro.

En el norte de Europa, Finlandia y Suecia constituyen otra posible línea de ampliación de la OTAN, una incorporación que aportaría grandes beneficios geoestratégicos y de potencial. Pese a todo, no vamos a analizar esta posibilidad, ya que consideramos que ambos Estados nórdicos no tendrían problema político, económico o militar para su integración en la organización. Además, al encontrarse ajenos a la influencia rusa, su incorporación no supondría, en ningún caso, el aumento de la tensión política con Rusia. Durante la Cumbre de Gales hubo un acercamiento positivo entre la organización y estos Estados, plasmado en la firma de un "host nation status agreement" con la OTAN (Wolff, 16 de septiembre de 2015, p. 1116).
Con respecto a las vías de ampliación de la OTAN sobre las que vamos a reflexionar —la balcánica, Cáucaso y Europa Oriental-, las posibilidades en ambos casos son dispares: si en el caso de los Balcanes, por un lado, la integración puede ser más pacífica y el margen de autonomía de dichos Estados con respecto a Rusia es aparentemente mayor, la zona caucásica y los Estados "colchón" de la frontera rusa occidental —Bielorrusia y Ucrania-, por otro, ofrecen una alta incertidumbre. Su membresía en la OTAN tendría un beneficio dudoso con respecto al alto riesgo de enfrentamiento, hasta sus últimas consecuencias, con Rusia.

Con respecto al primer supuesto, no cabe duda de que, a la postre, prácticamente todos los Estados balcánicos acabarán ingresando en la OTAN. En teoría, ello aportará un plus de estabilidad a la delicada región balcánica. Exceptuando el caso de Serbia (OTAN, 24 de noviembre de 2016) y el más espinoso de Kosovo, es plausible que la integración del resto de Repúblicas Balcánicas se dé en un futuro cercano con el beneplácito de los aliados europeos y, al menos, la no oposición furibunda de Rusia. De hecho, en el caso de Montenegro, ya se ha firmado el protocolo de Adhesión durante 2016, lo que le permite ser país observador hasta cuando se produzca su entrada definitiva al ratificarse dicho protocolo. Entretanto, Bosnia-Herzegovina continúa como Estado candidato.

Mayor es la incógnita con respecto a la posible incorporación de los países 
"tapón" de Europa Oriental fronterizos con Rusia: Bielorrusia y Ucrania (más su vecina Moldavia), así como la zona del Cáucaso más al sureste (en Eurasia), a caballo entre Occidente y Oriente. En este caso, hablamos de las repúblicas transcaucásicas fronterizas con Rusia y situadas entre el mar Caspio y el mar Negro: Georgia y Azerbaiyán, a las que habría que sumar Armenia, situada entre ambas.

De darse esta expansión, la OTAN trazaría un arco desde los Estados bálticos hasta los Estados transcaucásicos mencionados que dejaría a la organización a las puertas de la frontera rusa, que ya no contaría con países intermedios a modo de franja de protección. No obstante, parece difícil que Rusia acepte de buen grado una expansión semejante sobre sus reductos de Europa Oriental, de gran importancia estratégica y que considera una zona natural de influencia rusa.

Examinemos ahora cuál ha sido la relación de los Balcanes Occidentales con la OTAN. Tres nuevos Estados, Serbia, Montenegro y Bosnia-Herzegovina (llamados the new three), ingresaron en la Asociación para la Paz en 2006. La OTAN, como premisa, había establecido que estos demostrasen la voluntad de no dejar impunes a los principales responsables de las atrocidades cometidas en la Guerra de los Balcanes.

En el caso de Serbia, especialmente significativo por el nacionalismo exacerbado y la limpieza étnica durante el conflicto balcánico, el apoyo de la
OTAN se dio a cambio de la lucha contra la impunidad, la cooperación con el Tribunal Penal para la Antigua Yugoslavia y la captura de los principales sospechosos de crímenes de guerra: R. Karadzic (líder político serbobosnio, conocido con el sobrenombre del Carnicero de los Balcanes) y el general R. Mladic (OTAN, 2005, p. 7). La detención de ambos fue en 2008 (The New York Times, 2008) y 2011, respectivamente. En la actualidad, ambos criminales se encuentran cumpliendo condena en la Unidad de Detención de Naciones Unidas (United Nations International Criminal Tribunal for the Former Yugoslavia, 2016).

El principal obstáculo de entendimiento entre Serbia y la OTAN radica en la operación unilateral Fuerza Aliada de la OTAN en 1999 y sus últimas consecuencias: la declaración unilateral de independencia de Kosovo en 2008 por parte de las instituciones provisionales de su autogobierno. El denominado Plan Ahtisaari de la ONU, que pretendía dotar de un estatuto político a la provincia de Kosovo, fue muy criticado por la proposición de medidas claramente encaminadas a otorgar a la provincia un estatus político independiente, aunque tutelado por la comunidad internacional. El plan no salió adelante en el Consejo de Seguridad debido al veto ruso, pero la voz de este consejo fue irrelevante en este caso. Pese a las continuas protestas de Serbia o Rusia ante la inminente secesión, el Parlamento kosovar autoproclamó la independencia de Kosovo el 17 de febrero de 2008, siendo pos- 
teriormente reconocida por potencias occidentales de peso, especialmente por Estados Unidos y, en Europa, por Francia, Alemania, Reino Unido e Italia. La oposición a la independencia se había manifestado de forma meridiana por otros Estados pertenecientes a la UE y la OTAN, como España, Grecia y Rumania. Con dicha declaración de independencia, y el apoyo de muchos países mediante el reconocimiento del "nuevo Estado", se incumplía el respeto a la "soberanía e integridad territorial de la República Federativa de Yugoslavia", establecido en la Resolución 1244 del Consejo de Seguridad de Naciones Unidas (Consejo de Seguridad, 1999), que gestionaba el posconflicto tras la intervención de la OTAN.

La situación vino a enturbiarse aún más tras el sorprendente "Dictamen" (julio de 2010) emitido por el Tribunal Internacional de Justicia, a petición de la Asamblea General de Naciones Unidas (8 de octubre de 2008), en el que se debía dilucidar si la declaración unilateral de independencia emitida en el Parlamento kosovar se ajustaba o no al derecho internacional. Sin aportar una respuesta sustantiva, la Corte expresó que este tipo de declaraciones no estaban prohibidas ni permitidas por el derecho internacional, ya que responden a actos políticos internos que escaparían al control de este. Por posibles analogías que pudieran establecerse con regiones españolas donde existen partidos con aspiraciones independentistas (Cataluña y el País Vasco), la mejor doctrina española ha estado especialmente atenta a este "Dictamen", como lo demuestra el número monográfico de la Revista Española de Derecho Internacional, dedicado a la Opinión consultiva de la Corte Internacional de Justicia del 22 de julio de 2010 sobre la conformidad de la declaración unilateral de independencia de Kosovo con el Derecho internacional (véase Mangas Martín, 2011).

Kosovo se ha presentado, no obstante, como un caso especial o sui géneris de autodeterminación, porque es evidente que si el nuevo Estado no ha nacido desde una tabula rasa ni responde a un caso de descolonización de una ocupación o dominación extranjera, deberían haber prevalecido la intangibilidad de las fronteras y el respeto de la soberanía territorial de Serbia, principios que además se recogieron expresamente en la Resolución 1244 del Consejo de Seguridad (véase Mangas Martín, 2011, p. 102).

Por otro lado, si tomamos en consideración la denominada dimensión interna del derecho de autodeterminación, teóricamente aplicable a aquellas minorías cuyos derechos fundamentales no están siendo respetados por parte del Estado central y, por ende, existe discriminación y falta de representatividad democrática de estas en las estructuras estatales, llegando en casos extremos a una violación manifiesta de los derechos humanos, también habría que deducir que en el caso de Kosovo, y pese a la vulneración de derechos humanos que se constató en los momentos álgidos del conflicto serbio- 
kosovar, en la fecha de la declaración de independencia difícilmente tendría cabida el argumento de discriminación y opresión de una minoría étnica y socialmente diferenciada, como la albano-kosovar. Como ha especificado el profesor Jiménez Piernas, en dicho territorio

[...] ya se había superado la situación de graves violaciones de los derechos humanos padecida sobre todo entre 1996 y 1999, y en la fecha crítica de 2008, año de la declaración de independencia, Kosovo gozaba ya de una amplia autonomía o autogobierno. (2011, p. 47)

Con la ambigua respuesta del Tribunal Internacional de Justicia, Kosovo queda en un estatus irregular, pero políticamente proclive a consolidar de facto la independencia. Conforme a ello, podemos concluir, como afirma la profesora Mangas Martín, que en el dictamen se hacen primar "los intereses políticos geoestratégicos sobre el respeto del Derecho" (2010, s. p.).

La OTAN, por su parte, dada la crisis que había creado la falta de acuerdo entre los Estados aliados sobre Kosovo, evitó pronunciarse sobre el estatuto jurídico de Kosovo. En principio, se limitó a indicar que continuaría la presencia de la misión Kosovo Force (KFOR) en el territorio, para apoyar a la población y proteger la estabilidad de la región (OTAN, 18 de febrero de 2008). Es lógico que tras estos avatares —un bombardeo de 72 días de posi- ciones serbias en 1999 y la declaración de independencia de la provincia de Kosovo en 2008-, la población serbia y sus dirigentes no se hayan mostrado proclives al ingreso en la OTAN. De hecho, Serbia se declaró Estado militarmente neutral en 2007. Su decisión con respecto a la OTAN es colaborar activamente con ella $-\mathrm{y}$ así lo hace desde 2006 con la EAPC y el individual Partnership Action Plan (OTAN, 24 de noviembre de 2016)_, pero no integrarse en esta.

Por otra parte, la consolidación del Estado kosovar es un proceso inacabado. La oposición de muchos Estados a la ilegal intervención de la OTAN en Serbia en 1999 y a la (igualmente) ilegal independencia de Kosovo como Estado en 2008, planteada como una secesiónremedio por la violencia interétnica entre albano-kosovares y serbios, deja en una situación muy delicada a esta provincia. No en vano, desde círculos serbios, se llama a Kosovo: "the world's first NATO State" (Rodes, 2008, p. 17). La integración de Kosovo en la OTAN resultaría especialmente conveniente para algunas potencias (Osservatorio Italiano, 2010). A pesar de esto, es muy difícil que ciertos Estados de la UE y de la OTAN con problemas de independentismo, como es el caso de España, vayan a reconocer a Kosovo como un nuevo Estado. Es previsible, además, que otras potencias ajenas a ambas organizaciones nunca reconozcan a Kosovo como Estado, ya que dicho reconocimiento abriría una peligrosa puerta a los movimientos secesionistas, deseosos de utilizar po- 
líticamente este "caso especial" como un precedente legítimo para avalar sus argumentos. De hecho, la política intervencionista que Rusia ha emprendido desde 2008 (vide infra), otorgando apoyo abierto mediante el uso de la fuerza al independentismo en enclaves de antiguos Estados satélite con población rusa, debe entenderse - - según los analistas - como la respuesta de Rusia a la independencia de Kosovo. En efecto, tras la independencia de Kosovo, Rusia anunció que, en vista de lo sucedido, apoyaría la independencia de Osetia del Sur y Abjasia en Georgia, y de la República Srpska en Bosnia. Asimismo, consideraba apropiado apoyar la independencia de las regiones con población rusa de Transnistria (Moldavia) y Alto Karabaj (Azerbaiyán).

La fuerte controversia jurídica y política generada por la independencia de Kosovo ha propiciado que la comunidad internacional plantee la estatalidad de Kosovo como una situación "excepcional", que no debe sentar precedentes de independentismo en Estados estables y sin problemas interétnicos. Sin embargo, no cabe duda de que, para muchos grupos separatistas, Kosovo representa un modelo por seguir. Precisamente, por la falta del consenso que generaría la admisión de Kosovo como Estado en la OTAN, incluso entre los propios miembros de la Alianza, no parece factible que un Estado tan cuestionado vaya a ingresar en la organización (Rodes, 2008, pp. 17-18).
Montenegro vio entorpecida su incorporación a la OTAN en la Cumbre de Bucarest, debido a que, en su escaso recorrido como Estado democrático, no había llegado a cumplir con los estándares exigidos por la Alianza. Más tarde, a pesar de cumplir con dichos criterios democráticos, la OTAN decidió prolongar su espera en la Cumbre de Gales de 2014. Su admisión se decidió en febrero de 2016 (OTAN, 15 de febrero de 2016) y su entrada formal se produjo en junio de 2017. Pese a todo, y si bien desde su independencia en 2006 Montenegro comenzó a vincularse a la OTAN a través de la Asociación para la Paz, y de un modo más claro a través del MAP, gran parte de la población no se mostraba partidaria del ingreso en la OTAN. El rechazo de la población se debía, en parte, a que este Estado también fue objeto de bombardeos con la operación Fuerza Aliada, y a un hecho que no hay que menospreciar: un grueso importante de su población es de origen serbio. De hecho, el gobierno ha tenido protestas y manifestaciones en la capital, y desde el partido de la oposición se exigía un referéndum popular que avalase la entrada del país en la OTAN.

Rusia, por su parte, también ha expresado su descontento por la adhesión de Montenegro, dada la estrecha relación económica y turística que le une al pequeño país. Una vez más, Rusia ha remarcado su intención de responder a la OTAN por esta nueva incorporación (Navarro, 2015). Y es que, si bien Montenegro no puede aportar a 
la organización grandes efectivos en defensa, su posición en el Adriático le proporciona un alto valor estratégico.

Con respecto a Bosnia-Herzegovina, el principal lastre de este Estado es su batalla con la República Srpska, entidad regional autónoma del país donde vive un $49 \%$ de la población serbia. Recientemente, ha superado sus problemas y comenzó en 2010 las conversaciones para la adhesión.

Por lo que se refiere a la siguiente línea de ampliación señalada, abordaremos la situación de los países del Cáucaso, especialmente de Georgia. Es discutible si, geográficamente, podemos considerar a los países de esta región como europeos o, más bien, pertenecientes a Eurasia, y si tal ubicación puede afectar a la configuración atlántico-europea de la OTAN exigida en el artículo 6 del TAN.

Georgia, al igual que Ucrania (vide infra), manifestó muy pronto su deseo de integrarse en la OTAN; concretamente, desde 2002, cuando se gestaba la Revolución de las Rosas (2003) que expulsó de forma pacífica al presidente Shevardnadze en favor de un gobierno prooccidental que abogaba por reformas democráticas. Ambos Estados se encontraron en cabeza de lista para engrosar las filas de la Alianza durante la Cumbre de Bucarest de 2008. Su futura integración había sido avalada políticamente desde el propio Congreso de los Estados Unidos (François-Poncet et ál., 2006-2007, p. 12), que aprobó en marzo de 2007 un documento que apoyaba la ampliación ("NATO Freedom Consolidation Act"), con el presidente Bush como su principal valedor (Kramer, 2015, p. 4). Pero la relación con Rusia dificultó hasta extremos insospechados tal voluntad, ya que Putin, con motivo de la cumbre, expresó abiertamente que los asuntos de seguridad e intereses políticos rusos, especialmente en el espacio possoviético - pero no solamente allí - no podían seguir siendo ignorados por la OTAN (Kramer, 2015, p. 5). De hecho, Rusia amenazó a la OTAN sobre las posibles consecuencias de cruzar la "línea roja" e integrar sin contemplaciones a Georgia y Ucrania en la organización (Europa Press, 4 de abril de 2008).

La situación política era bastante delicada como para no pensarlo dos veces: incesantes advertencias rusas, un debate candente sobre el escudo antimisiles en las fronteras polaca y checa, $y$, dentro de la propia Alianza, una fuerte oposición por parte de algunos miembros —encabezados por Francia y Alemania- a la futura integración de Georgia y Ucrania (La Nación, 2008). Finalmente, ni Ucrania ni Georgia accedieron al MAP, por lo cual carecieron de un itinerario preciso de cara a la adhesión (Lazarevic, 2009, p. 45). La OTAN les tendió la mano formalmente al incluirles en los programas anuales nacionales (que se desarrollan habitualmente dentro del MAP), y así salvó la promesa de adhesión hecha por parte de Estados Unidos, pero sin llegar a materializar un compromiso claro en caso de que cumplieran los estándares requeridos como aspirantes. Se trata- 
ba, por tanto, de una "alteración del objetivo intrínseco del MAP" (p. 45), que dejaba en el limbo institucional a ambos Estados. En el caso de Georgia, además, podía conllevar cierto riesgo político, como el futuro rechazo hacia Occidente por parte del país caucásico (El Diario, 2013), pese a que el apoyo o la simpatía hacia la OTAN siempre han sido mucho más amplios y consolidados que en Ucrania.

La vocación de Georgia de integrarse en la Alianza Atlántica y la declaración de independencia de Kosovo en febrero de 2008 constituyeron un acicate de primer orden para una respuesta rusa ante lo que consideraba un "desafío" por parte de la exrepública soviética y de la propia OTAN. Tal respuesta consistió en el apoyo ruso a la independencia de determinados enclaves de Georgia con población rusa y afán independentista desde los años noventa: Abjasia (limítrofe con el mar Negro) y Osetia del Sur o Alta Osetia (fronterizo con Rusia). De hecho, la inestabilidad política de ambas regiones constituyó el principal impedimento para la admisión de Georgia en la OTAN.

Tras la independencia de Georgia en 1991, Osetia del Sur y Abjasia comenzaron un pulso independentista que se saldó con elecciones no reconocidas por Georgia. En 2006, las tensiones condujeron al gobierno de Saakashvili a desplegar el ejército para controlar y revertir el estatus de independencia de facto instaurado en ambas regiones. Pese a que durante ese año el ejército georgiano había logrado controlar parte del territorio tomado por los independentistas y había empujado al gobierno de estos hacia la Alta Abjasia, la situación cambió en 2008, cuando estalló un conflicto abierto con los independentistas, con Rusia como telón de fondo. Los rebeldes de Osetia fueron ayudados por las fuerzas rusas. Del mismo modo, Abjasia se vio apoyada por Rusia y otros grupos prorrusos que apoyaban la independencia, como chechenos y cosacos, que lograron vencer al ejército georgiano. Finalizó así un conflicto que se saldó con las declaraciones de independencia de estos enclaves.

Las fuerzas rusas que ayudaron a Osetia y Abjasia previamente se encontraban desplegadas como fuerzas de paz, en tanto que otros contingentes, sin justificación alguna, habían sido enviados por Moscú hacia dichas regiones con el argumento de ayudar en distintas tareas, en lo que a todas luces se mostraba como una maniobra de injerencia externa (OTAN, 3 de junio de 2008). De hecho, Rusia fue el primer Estado en reconocer la independencia de Osetia del Sur y Abjasia, fuertemente criticada por la UE, Estados Unidos y la propia OTAN, que a través de comunicados oficiales advirtió a Rusia del "error" de otorgar tal reconocimiento (OTAN, 26 de agosto de 2008).

Tras el conflicto, el entonces presidente de turno de la UE, Nicolas Sarkozy, realizó labores de mediación y llegó a un acuerdo el 13 de agosto con Moscú y Tiflis (Plan de Medvédev-Sarkozy o acuerdo de los seis puntos), que dio 
paso a la misión de observación de la UE (European Union Monitoring Mission in Georgia [EUMM]). El presidente de Georgia reclamó con urgencia el ingreso de su país en la OTAN. No en vano, el 19 de agosto de 2008 se constituyó la Comisión OTAN-Georgia, con el objetivo de apoyar y consolidar la estabilidad política del país, revisar el proceso de futura adhesión a la OTAN y cOoperar para su recuperación tras el conflicto (OTAN, 15 de septiembre de 2008).

Como se ha dicho, aunque el deterioro de las relaciones con Rusia comenzó unos años antes, el conflicto de Georgia en 2008 fue un punto de "no retorno" en el diálogo OTAN-Rusia (Priego, 2014). De hecho, la OTAN suspendió temporalmente las relaciones con Rusia, manifestando su no reconocimiento a los territorios independizados y su apoyo a la soberanía e integridad territorial georgianas. La acción de la OTAN no podría haber ido más allá, al no constituir Georgia un Estado miembro. Y sería poco realista negar que la situación territorial de Georgia - ya enquistada - no iba a constituir un serio impedimento para su total integración en la Alianza. De hecho, a pesar del esfuerzo que el Estado georgiano ha demostrado en la reforma de sus fuerzas armadas, su entrada en la OTAN no parece factible por el momento.

Durante los últimos años no se ha percibido que la OTAN esté absolutamente dispuesta a recibir, sin ambages, a Georgia. Las imprevisibles consecuencias que ello podría tener con Rusia son el principal freno. En la Cumbre de Gales de 2014 se evitó aludir a su posible adhesión. En la "Declaración final" de la cumbre no existe referencia alguna sobre el futuro de Georgia como miembro; tan solo se aprobó un "paquete de medidas" que indirectamente apoyaba una eventual integración. En la última declaración de ministros de Asuntos Exteriores sobre la política de puertas abiertas, se han paliado los silencios de la cumbre respecto al proceso de ampliación, alentando a Georgia a seguir cooperando con la OTAN y constatando que, prácticamente, cuenta con todos los requisitos necesarios para la integración (OTAN, 2 de diciembre de 2015, párrafos 10-13).

En la misma línea, la más reciente Cumbre de Varsovia en 2016 ha continuado sin ofrecer el MAP a este viejo aspirante. De hecho, en la "Declaración final" no se mencionó de forma palmaria una futura adhesión de Georgia a la OTAN; únicamente, se reitera que tiene abiertas las puertas de la Alianza. La "Declaración" le dedica una notable atención y agradece al Estado caucásico la colaboración con misiones de la OTAN (especialmente por su alta aportación de tropas en Afganistán), y se presta a otorgarle un apoyo especial. Dicho apoyo se ha traducido en un refuerzo de la cooperación militar con Georgia, al tiempo que la Alianza condena y exige de forma explícita a Rusia que revierta la situación que ha creado en Osetia del Sur y Abjasia (OTAN, 9 de julio de 2016, párrafos 111-113). 
De este modo, la idea que la OTAN parece enviar a Georgia es que mantenga la confianza y continúe en la lista de espera hasta que se den las circunstancias políticas oportunas que le permitan acceder a la organización como Estado miembro. La propia Georgia parece consciente de que esta esperanza es, por el momento, todo lo que la OTAN puede ofrecerle (Menabde, 2016). De esta manera, a pesar de la envidiable situación geoestratégica de Georgia, ubicada entre el mar Negro, Turquía, Armenia, Azerbaiyán y Rusia, el escollo de su complicada situación territorial —con dos regiones "independizadas" con la ayuda rusa (Wolff, 2015, p. 1117) — va a ser difícil de salvar. Esto, especialmente, si algunos Estados aliados de peso dentro de la OTAN son reticentes a su integración, conscientes de que cualquier problema entre Rusia y Georgia implicaría una eventual activación de la solidaridad aliada a través del artículo 5 del TAN. Si bien Estados Unidos ha demostrado siempre un firme apoyo a la candidatura georgiana, otros miembros (Alemania, Polonia y Francia) se oponen a esta. Las circunstancias internas de este país aspirante y la sombra rusa que se cierne sobre él tendrían que cambiar mucho para que la Alianza Atlántica manifestase la unidad de criterio con respecto a su futura integración en la organización, pues comprometería en exceso las relaciones con Rusia (Dempsey, 2015, p. 1) y, finalmente, la estabilidad regional.

\section{Ucrania, entre el deseo atlantista y la influencia rusa}

Ucrania, el segundo país más grande de Europa, se caracteriza por un importante potencial geoestratégico (limítrofe con siete Estados y el mar Negro, y principal zona de tránsito del gas ruso a Europa), humano (más de 46 millones de habitantes) y económico. Al día de hoy, este enorme Estado representa la línea roja de la ampliación de la OTAN. Aunque existen distintos factores que alejarían a Ucrania de la OTAN, el principal es el siguiente: "Ukraine's membership in NATO for Russia is, as has been very clearly put by the Russian side, 'out of question'" (Lazarevic, 2009, p. 48). Es decir, para Rusia es indiscutible una Ucrania fuera de la OTAN.

Situado entre Oriente y Occidente y cuajado de historia e identidad propias, Ucrania es un territorio que nunca ha conocido, hasta el final de la Guerra Fría, la independencia política (Requena del Río, 2014, p. 1 y ss.). No obstante lo anterior, los tentáculos de la influencia rusa siguen poniendo en entredicho que esa independencia sea efectiva. No es de extrañar, por tanto, que desde 1991 Ucrania trate de encontrar su lugar político y que se halle inmerso en un proceso de búsqueda de identidad entre distintas fuerzas, fundamentalmente aquellas tendentes a Occidente y las proclives a Rusia. 
El acercamiento de Ucrania a las instituciones occidentales es visto por Rusia como una pérdida intolerable de su espacio defensivo natural en la frontera occidental (Blank y Huessy, 2015), al constituir Ucrania el "Estado tapón" (buffer zone) por excelencia entre Rusia y Europa. Una Ucrania inserta en las instituciones occidentales es percibida por el Kremlin como una de las más importantes pérdidas de poder en el espacio postsoviético, un espacio que trata de mantener y afianzar a través de diversas organizaciones de carácter económico, político y militar (Comunidad de Estados Independientes, Unión Euroasiática y Organización del Tratado de Seguridad Colectiva). A estas consideraciones se unen otras razones de carácter histórico y cultural. Los lazos que unen a ambos Estados son realmente fuertes, tanto por origen como por la situación demográfica actual, ya que la población rusa en Ucrania constituye el segundo grupo más importante del país, de modo que "para Rusia la defensa de los veinte millones de rusos étnicos que quedaron fuera de sus fronteras tras la disolución de la URSS es un interés vital" (Ruiz, 2014).

Por lo que se refiere a razones geoestratégicas más concretas en la relación Rusia-Ucrania, la península de Crimea, con la importante base naval rusa de Sebastopol en el mar Negro, constituyó desde la independencia de Ucrania una pieza clave de los desencuentros entre ambos Estados. Crimea, esta península de alto valor estratégico, que da salida a Rusia al mar Mediterráneo, "ha sido siempre un capítulo no cerrado en las relaciones bilaterales ya que una importante parte de los rusos la consideran suya" (Dolya, 2016, p. 1). No en vano fue Nikita Kruschev quien la cedió a la República Ucraniana en 1954, y tal decisión fue revocada por Rusia tras la disolución de la URSS, alegando la irregularidad de la cesión y el predominio de población rusa en dicha península (Dolya, 2016, p. 1). Desde esa pretensión de propiedad legítima, a Rusia no le agradaba tener que negociar con Ucrania la permanencia de la flota rusa en la base naval. En el último acuerdo entre Putin y Yanukovich (presidente de Ucrania, 2010-2014), se garantizó la permanencia rusa en Sebastopol hasta el año 2042 (Requena del Río, 2014, p. 5). Por ello, pese al modus operandi ruso en la anexión de Crimea (vide infra), el objetivo de Rusia no resultaba inesperado teniendo en cuenta la historia y la constante reivindicación del Kremlin sobre dicha península.

Como es sabido, la reciente crisis de Ucrania y la reacción de Rusia no fueron provocadas por una hipotética ampliación de la OTAN a este Estado, sino por el acercamiento del gobierno ucraniano a la UE, materializado en un posible Acuerdo de Asociación y Libre Comercio. La opción por dicha asociación con la UE coartaba el proyecto de Unión Económica Euroasiática que Moscú estaba pergeñando para 2015, con la finalidad de agrupar a las exrepúblicas soviéticas bajo un mercado común, por lo que la reacción rusa no se hizo esperar (Walker, 2015, p. 5). 
La presión rusa se tradujo en diversas medidas, algunas coactivas, como la amenaza de represalias económicas por parte de Rusia, y otras "seductoras", como la bajada de precios del gas. Dicha presión obtuvo sus frutos con Armenia y con la propia Ucrania, que abrazaron a Rusia como mejor postor y rechazaron a la UE (Ruiz, 2014). Fue en este momento cuando entró en juego la revolución del Euromaidán, las intensas protestas civiles en otoño de 2013 que se sucedieron en el país tras el giro prorruso por parte de Yanukovich. Dichas protestas agruparon fuerzas de diversa índole y se hicieron cada vez más violentas, y finalizaron con la deposición de Yanukovich por parte del Parlamento. Yanukovich se vio obligado a huir del país, en tanto que la convocatoria de nuevas elecciones otorgó el poder a Poroshenko en junio de 2014. Antes de ello se produjo uno de los episodios más controvertidos de lo que parecía ser un estertor de la Guerra Fría: la anexión de Crimea a la Federación Rusa, mediante un referéndum celebrado en marzo de 2014, en el que por una abrumadora mayoría (96,6\%) la población de Crimea, rusa casi en su totalidad, decidía romper unilateralmente con Ucrania e integrarse en la Federación Rusa con el nombre de República de Crimea y Sebastopol, y ampararse así en un pretendido derecho a decidir su autodeterminación como pueblo. Rusia actuó en un tiempo récord para organizar un referéndum ilegal y proteger lo que consideraba un interés vital en Ucrania (Dolya, 2016, p. 3). El resultado de la consulta estaba asegurado, ya que a la mayoría de población rusa se unió una incesante entrada en Crimea de fuerzas rusas, militares y paramilitares, que fueron controlando y pertrechando el territorio de la península desde febrero.

El fuego cruzado de acusaciones entre Rusia y Occidente no se hizo esperar; frente a las condenas de ilegalidad del proceso y de la guerra híbrida que estaba teniendo lugar, Rusia sacó a relucir el también controvertido caso de Kosovo y el quebrantamiento de la promesa de respetar la integridad territorial serbia (Levy, 2014; Rizzi, 2014).

Seis días más tarde, Putin validó ante la Duma, con toda pompa y boato, la anexión de Crimea a Rusia. Se basa no solo en los lazos históricos que unen a ambos territorios, considerando que se trata más bien de una "reunificación". Al referirse a los argumentos jurídicos, Putin hizo hincapié, con gran ironía, en el ambiguo dictamen sobre Kosovo emitido por el Tribunal Internacional de Justicia, en el que este último consideró que "el derecho internacional no contiene ninguna prohibición aplicable a la declaración de independencia". Además de ello,

[...] reprodujo dos frases del memorando fechado el 17 de abril de 2009 que EE. UU. presentó a dicho Tribunal en relación a Kosovo: "las declaraciones de independencia pueden contradecir, y con frecuencia así sucede, las leyes internas; sin embargo, ello no significa que se esté violando el derecho internacional". (Fernández, 18 de marzo de 2014) 
Las condenas internacionales, también desde Naciones Unidas (Asamblea General de las Naciones Unidas, 2014), y las diversas sanciones institucionales (fundamentalmente desde la UE) y bilaterales por parte de las principales potencias occidentales no evitaron que el mismo modus operandi de apoyo soterrado a los separatistas por parte de Rusia se haya repetido en las regiones orientales separatistas de Donetsk y Lugansk, que se han proclamado repúblicas populares independientes bajo el amparo ruso. El control de estos territorios significa para Ucrania un espinoso problema interno e internacional.

Los acuerdos de Minsk (2014 y 2015) no han logrado finalizar realmente el conflicto entre los rebeldes separatistas y el gobierno ucraniano. Pese a que el conflicto tiene menor resonancia informativa que antes, las bajas continúan en aumento (casi cien mil), la lucha no ha cesado y las víctimas civiles (en torno a dos mil) y violaciones de derechos humanos están a la orden del día. Ucrania se halla, por tanto, inmersa en un conflicto civil de baja intensidad que no tiene visos de ser resuelto en breve.

Bajo estas circunstancias, la posición internacional de Ucrania se complica. Considerada un "near abroad" imprescindible para Rusia, ya se encontraba en una posición realmente compleja con respecto a una posible adhesión a la OTAN. En este momento, las nuevas circunstancias de inestabilidad y conflicto en las zonas orientales, derivadas de la política de hechos consumados por parte de Rusia, dejan la posible candidatura en unas condiciones menos favorables aún.

Si bien el futuro es incierto, podemos barajar varias hipótesis de cara a una futura adhesión de Ucrania a la OTAN. En primer lugar, la candidatura de Ucrania podría quedar bloqueada o blindada, dependiendo de cómo se sucedan los acontecimientos. Posiblemente hablemos de bloqueo o enquistamiento temporal si, a medio plazo, la situación de independentismo de la zona oriental se resolviese favorablemente para la integridad territorial de Ucrania y el Estado fuera capaz de mantener una independencia real frente al gigante ruso. Ello es improbable si Ucrania no aminora la dependencia energética (especialmente de gas y electricidad), así como también la económica, con respecto a Rusia. Para ello tendría que recibir un fuerte respaldo económico por parte de la UE, especialmente de Francia y Alemania, cuya dependencia de la energía rusa también determina notablemente su política exterior con el Kremlin. También podríamos barajar la alternativa de bloqueo temporal de la candidatura ucraniana a la Alianza en el caso opuesto, es decir, si dichas regiones orientales finalmente acabasen desgajadas del país y anexionadas a Rusia. En este caso, la parte occidental posiblemente tendría la oportunidad de decidir su futuro institucional sin la sombra de la amenaza rusa, aunque quedase mermada territorialmente. 
No obstante, tendríamos que hablar de blindaje o acorazamiento si la permanencia de tropas rusas - regulares e irregulares- y la ayuda logística por parte de Moscú a los rebeldes separatistas se prolongase de forma indefinida en la zona oriental, con la consecuencia o el resultado de dos conflictos enquistados que impidieran un control pleno y efectivo de Ucrania sobre Donetsk y Lugansk. En ambas regiones, pese a haberse autoproclamado Repúblicas independientes, la mayor parte del territorio se encuentra bajo el gobierno ucraniano; pero se trata de un pulso incesante que puede verse revertido en cualquier momento. Las hipótesis son variadas y unas resultan más plausibles que otras. El blindaje a través del apoyo al separatismo en las regiones del este de Ucrania y la generación de una constante inestabilidad en dichas zonas desde Rusia parecen, por el momento, las conjeturas más acertadas.

Hasta hace poco tiempo se podía considerar que Georgia y Ucrania, a pesar de sus similitudes como candidatos a la integración en la OTAN ${ }^{1}$, eran casos distintos. Precisamente, y sin que ninguno de los Estados fuese un candidato claro debido a la presión rusa, la diferencia fundamental radicaba en el problema territorial que lastraba a Georgia desde 2008 (Lazarevic, 2009, pp. 47 y ss.). Actualmente, sin embargo, ambos Estados conllevan las mis- mas cargas. Las consecuencias de una posible ampliación a Ucrania serían difíciles de asumir por parte de la OTAN, más que hace unos años. La Alianza tendría que estar dispuesta a afrontar la reacción rusa en todos los niveles, desde las relaciones diplomáticas hasta una posible activación del artículo 5 del TAN para defender la integridad territorial y la independencia política de un Estado miembro. Es obvio que la adhesión de Ucrania podría tener un alto coste político y militar para el resto de los miembros.

Por si estos motivos no fueran de peso, tampoco hay que menospreciar que, pese a que la OTAN siempre ha mantenido un interés especial con respecto a Ucrania, como lo manifestó al otorgar un estatus diferenciado a este país con la "Carta sobre una asociación distintiva entre la OTAN y Ucrania" en 1997 (OTAN, 11 de julio de 2017), lo cierto es que el entusiasmo de la población ucraniana por la OTAN no se ha visto correspondido en la misma medida. Además de ello, los respectivos presidentes de Ucrania desde la independencia tampoco han mostrado una línea coherente con respecto al futuro de su política exterior, y sí devaneos entre Rusia y Occidente. A pesar de que la reciente decisión de Poroshenko en 2014 de abolir el estatus de no alineado del país ha contribuido notablemente a despejar dichas dudas y señalar claramente la vocación

1 Es decir, tuvieron Revoluciones de Colores, que se instauraron en 2003 y 2004, respectivamente; se consideran gobiernos democráticos; constituyen zonas de paso del gas y petróleo rusos, y, por último, son zonas de influencia que Rusia no desea perder. 
prooccidental de Ucrania, su destino político, por el momento, parece estar más ligado a la determinación política de Rusia que a su propia voluntad.

En las próximas décadas, el rumbo de la política exterior rusa con Europa y el resto del mundo podría cambiar. No obstante, esta es una variable que, por el momento, no podemos contemplar. Y menos aún en la "era Putin", con su remarcado acento autoritario. Ucrania se configura como una pieza clave del futuro devenir ruso. Tal y como pronosticó con acierto Brzezinski, todavía no hemos visto el momento "definitorio" de las relaciones entre Rusia y Europa. Si Rusia finalmente acepta los lazos de Ucrania con las instituciones occidentales, esto significará que habrá aceptado su propia caracterización como potencia europea, mientras que si los rechaza seguiría manteniendo su "identidad y existencia solitariamente 'euroasiáticas'” (Brzezinski, 1998, p. 126). Ucrania, por tanto, inclinará la balanza de un futuro ruso dentro o fuera de Europa. Por ello, podemos calificar al gigante ucraniano como la piedra angular por excelencia entre Rusia y Occidente.

\section{Conclusión}

La ampliación de la OTAN parece haber tocado techo, al menos en la frontera occidental rusa, donde únicamente los Estados bálticos, en la actualidad preocupados por posibles incursiones rusas, y Finlandia, escapan de la órbita de influencia rusa. Bielorrusia, Ucrania, Moldavia y Georgia, con conflic- tos congelados creados por Rusia, no resultan candidatos fáciles de admitir por el resto de los miembros.

El deseo de engrosar las filas de la OTAN por parte de los Estados que se zafaron del poder soviético tras décadas de dominio político tuvo como resultado dos importantes rondas ampliadoras hacia Europa Central y Oriental. En la balanza de las primeras rondas ampliadoras de la Posguerra Fría (1999 y 2002), venció la geopolítica sobre otras consideraciones de los Estados candidatos (cumplimiento de criterios exigidos por la OTAN, aportación efectiva de recursos, etc.), un hecho supuestamente debido a la intención de la OTAN de unir la Europa fracturada entre el Este y el Oeste. Pero Rusia, lógicamente, nunca ha percibido de este modo su pérdida de influencia en el espacio postsoviético, y la actitud del Kremlin, irremediablemente, ha condicionado en mayor o menor medida el proceso ampliador (Martínez Laínez, pp. 52-57).

Moscú, en los últimos años, ha planteado sin lugar a equívocos el espacio de influencia en su "near abroad" que no está dispuesto a perder bajo ningún concepto. Las líneas rojas han sido puestas en Georgia y, especialmente, en Ucrania. En ambos casos, se ha dado una nueva vuelta de tuerca en la relación de la OTAN con Rusia, tras el uso de la fuerza empleado por Rusia para "proteger" sus intereses y población rusa en ambos Estados. Se trata de una política exterior agresiva que atiende al principio del equilibrio de 
poder más descarnado, descrito por Kelsen así: "a principle of political convenience" (1948, p. 42). Tras la guerra de Georgia en 2008 (Europa Press, 19 de agosto de 2008), así como después del conflicto de Ucrania en 2014 (Fernández, 4 de septiembre de 2014), la OTAN ha experimentado un punto de inflexión en el entendimiento con Rusia, y ha llegado a un límite que, por el momento, no puede rebasar con respecto al proceso ampliador.

Repensar la ampliación y sus beneficios estratégicos parece ser la idea que preside la nueva estrategia política de la Alianza Atlántica (Wolff, 2015, p. 1114). Coincidimos con Rubio Plo en lo siguiente: "El principal test de seguridad colectiva pasa no solo por la defensa, sino además por la capacidad de ampliación de la Alianza, pero esta parece haber tocado fondo hace tiempo" (2014, p. 2). Es evidente que Estados aliados de peso en Europa consideran oportuno mantener una relación positiva, en la medida de lo posible, con Rusia. No en vano, Francia y Alemania reiteradamente han rechazado la candidatura de Ucrania y Georgia a la OTAN. Si en el caso de Georgia las posibilidades de incorporación a la Alianza parecen mayores (y ello preocupa especialmente a Rusia), con respecto a Ucrania la coyuntura poco propicia para la admisión de este Estado como miembro de la OTAN admite pocas dudas. De hecho, si la respuesta a la anexión de Crimea por parte de Occidente no ha sido más contundente, las razones parecen obvias: también se necesita a Rusia para luchar contra el terrorismo del Daesh, ya sea en Afganistán o en Siria, pese a las diferencias de la coalición occidental con Moscú en este último país. Este aspecto fue, sin duda, tomado en cuenta por Estados Unidos bajo la administración de Obama.

Las nuevas ampliaciones de la OTAN posiblemente traten de mantener la estabilidad internacional, el equilibrio y el mayor beneficio posible para todos los Estados de la zona euroatlántica. Es obvio que los desencuentros y las diferentes posturas políticas con Rusia son y serán, en ocasiones, irreconciliables. No obstante, los Estados aliados tratan de evitar romper la baraja diplomática y llegar a límites de consecuencias indeseables para todos los actores implicados. En las actuales circunstancias de impasse con respecto a la ampliación, parece más adecuado que la OTAN mantenga, en la medida de lo posible, una política de diálogo con Rusia, sin dejar de procurar una estrecha cooperación con los Estados que deseen pertenecer en el futuro a la alianza defensiva más fuerte del planeta. Buscar el momento propicio con Rusia, mientras se ejerce la seducción del soft power con los Estados candidatos, no responde a una claudicación, sino a una jugada inteligente y responsable en la partida de ajedrez de las relaciones diplomáticas.

\section{Referencias}

Asamblea General de las Naciones Unidas. (27 de marzo de 2014). 
Resolución 68/262. Integridad territorial de Ucrania. Recuperado de https://undocs.org/es/A/RES/68/262

Asamblea General de las Naciones Unidas. (8 de octubre de 2008). Proyecto de resolución 63.L.2. Solicitud de una opinión consultiva de la Corte Internacional de Justicia respecto de si la declaración unilateral de independencia de Kosovo se ajusta al derecho internacional. Recuperado de https://undocs.org/ es/A/63/L.2

Bilefsky, D. (23 de julio de 2008). Karadzic Arrest Is Big Step for a Land Tired of Being Europe's Pariah. The New York Times. Recuperado de https:// www.nytimes.com/2008/07/23/ world/europe/23serbia.html

Blank, S. y Huessy, P. (3 de febrero de 2015). NATO Enlargement and Ukraine. Myth vs. Facts, Gatestone Institute. Recuperado de http:// www.gatestoneinstitute.org/5164/ russia-ukraine-nato-enlargement

Brzezinski, Z. (1998). El gran tablero mundial. La supremacía estadounidense y sus imperativos geoestratégicos. Barcelona: Paidós.

Caracuel, M. (2004). Los cambios de la otan tras el fin de la Guerra Fría. Madrid: Tecnos.

Consejo de Seguridad de la OTAN. (1999). Resolution 1244. Recuperado de http://www.nato.int/kosovo/docu/u990610a.htm

Corte Internacional de Justicia (CII) (17 de octubre de 2008). Conformité au droit international de la déclaration unilatérale d'indépendance des institutions provisoires d'administration autonome du Kosovo. Recuperado de https://www.icj-cij.org/files/ case-related/141/15699.pdf

Corte Internacional de Justicia (CIJ) (17 de noviembre de 2008). Application of the Interim Accord of 13 September 1995 (the former Yugoslav Republic of Macedonia v. Greece). Recuperado de https://www. icj-cij.org/en/case/142

Dempsey, J. (5 de agosto de 2015). Who's afraid of NATO Enlargement? Carnegie Europe. Recuperado de http://carnegieeurope.eu/ strategiceurope/?fa $=60945$

Diez años después, Georgia relega al olvido la Revolución de las Rosas. (23 de noviembre de 2013). El Diario. Recuperado de http:// www.eldiario.es/politica/despuesGeorgia-relega-Revolucion-Rosas_0_199830168.html

Dolya, A. (22 de febrero de 2016). L'annexion de la Crimée: leçons pour la sécurité européenne. Foundation Robert Schuman. Recuperado de https://www.robert-schuman. eu/fr/questions-d-europe/0382l-annexion-de-la-crimee-leconspour-la-securite-europeenne

EE. UU. pedirá "revisar" la cooperación entre la OTAN y Rusia tras la invasión de Georgia. (19 de agosto de 2008). Europa Press. Recuperado de https://www.europapress.es/ internacional/noticia-eeuu-pedirarevisar-cooperacion- otan-rusia-in- 
vasion-georgia-20080819090104. html

Ferguson, N. (enero-marzo de 2010). El año en que el mundo cambió de verdad. La Vanguardia Dossier (34), 16-20.

Fernández, R. (18 de marzo de 2014). Putin firma la anexión de Crimea a Rusia. El País. Recuperado de https://elpais.com/ internacional/2014/03/18/actualidad/1395125826_603105.html

Fernández, R. (4 de septiembre de 2014). Rusia advierte en contra de que Ucrania forme parte de la OTAN. El País. Recuperado de https://elpais. com/internacional/2014/09/04/ actualidad/1409838616_104186. html

Fundación Instituto de Cuestiones Internacionales y Política Exterior (Incipe) y Fundación Real Instituto Elcano (2008). La Cumbre de Bucarest: preparando el camino para el 60. $\mathrm{o}$ aniversario de la OTAN. Madrid: Fundación Instituto de Cuestiones Internacionales y Política Exterior (Incipe), Fundación Real Instituto Elcano. Recuperado de http://realinstitutoelcano.org/wps/wcm/con nect/213e8f004f0196208ba6ef31 70baead1/Cumbre_Bucarest_\%20 OTAN_2008.pdf?MOD=AJPERES

François-Poncet, J. Branger, J-G. y Rouvière, A. (2006-2007). Rapport d'information fait au nom de la commission des Affaires étrangères, de la défense et des forces armées sur l'évolution de l'OtAN. Recupera- do de http://www.senat.fr/rap/r06405/r06-4051.pdf

Jiménez, C. (2011). Los principios de soberanía e integridad territorial y de autodeterminación de los pueblos en la opinión consultiva sobre Kosovo: una oportunidad perdida. Revista Española de Derecho Internacional, 63(1), 29-54. Recuperado de http://bibliotecaculturajuridica. com/biblioteca/arxius/PDF/REDI Vol._LXIII_1_2011/02_Jimenez_digital.pdf

Kelsen, H. (1948). Collective security and Collective defense. American Journal of International Law, 42(4), 783-796.

Law, D. (1999). Why Spain should have been NATO's last member. En C. David y J. Lévesque (eds.), The future of NATO. Enlargement, Russia, and European Security (pp. 35-50). Quebec: McGill University.

Lazarevic, D. (2009). NATO Enlargement to Ukraine and Georgia: Old Wine in New Bottles? Connections, The Quarterly Journal, 9(1), 29-66.

Levy, B-H. (17 de marzo de 2014). Frente a Putin, no renunciemos a la verdad. El País. Recuperado de https:// elpais.com/elpais/2014/03/14/opinion/1394812091_721685.html

Mangas, A. (26 de julio de 2010) Kosovo: abierta la caja de Pandora. El Mundo. Recuperado de https://aracelimangasmartin.com/ wp-content/uploads/2017/02/Kosovo-abierta-la-caja-de-PandoraEl-Mundo-26-de-julio-de-2010.pdf 
Mangas, A. (2011). Kosovo y la Unión Europea: una secesión planificada. Revista Española de Derecho Internacional, 63(1), 101-123. Recuperado de http://bibliotecaculturajuridica.com/biblioteca/arxius/ PDF/REDI_Vol._LXIII_1_2011/05_ Mangas_digital.pdf

Martínez, F. (diciembre de 2013). Moscú busca su lugar. Revista Española de Defensa, 26(301), 52-57. Recuperado de http://www.ieee.es/Galerias/fichero/OtrasPublicaciones/ Nacional/RED_301.pdf

Menabde, G. (18 de julio de 2016). Georgia receives 'Maximum Possible' at NATO's Warsaw Summit. Eurasia Daily Monitor 13(129). Recuperado de https://jamestown. org/program/georgia-receivesmaximum-possible-at-natos-warsaw-summit/

Müller, E. (11 de febrero de 2007). Putin revive el fantasma de la Guerra Fría en Múnich con un ataque frontal a eE.uU. El Diario Vasco. Recuperado de http://www.diariovasco.com/prensa/20070211/ mundo/putin-revive-fantasma-guerra_20070211.html

Navarro, B. (3 de diciembre de 2015). La OTAN irrita a Rusia al abrir sus puertas a Montenegro. La Vanguardia. Recuperado de http:// www.lavanguardia.com/internacional/20151203/30556452216/otanrusia-montenegro.htm

Organización del Tratado del Atlántico Norte (OtAN). (3 de septiembre de 1995). Study on NATO Enlargement.
Recuperado de http://www.nato.int/ cps/en/natolive/official_texts_24733. htm? selectedLocale $=$ en

Organización del Tratado del Atlántico Norte (OtAN). (18 de febrero de 2008). Statement by the North Atlantic Council after Kosovo's Declaration of Independence). Recuperado de http://www.nato.int/ docu/pr/2008/p08-025e.html

Organización del Tratado del Atlántico Norte (OtAN). (3 de abril de 2008). Bucharest Summit Declaration. Recuperado de http://www.nato.int/ cps/en/natohq/official_texts_8443. htm?selectedLocale $=$ en

Organización del Tratado del Atlántico Norte (OtAN). (3 de junio de 2008). NATO Secretary General's statement on the Deployment of Russian RaiIway Troops into Georgia. Recuperado de http://www.nato.int/docu/ pr/2008/p08-076e.html

Organización del Tratado del Atlántico Norte (OtAN). (26 de agosto de 2008). Statement by the Secretary General of NATO on the Russian recognition of Abkhazia and South Ossetia. Recuperado de http:// www.nato.int/docu/pr/2008/p08107e.html

Organización del Tratado del Atlántico Norte (otan). (27 de agosto de 2008). Statement by the North Atlantic Council on the Russian recognition of South Ossetia and Abkhazia regions of Georgia. Recuperado de http://www.nato.int/docu/pr/2008/ p08-108e.html 
Organización del Tratado del Atlántico Norte (OTAN). (15 de septiembre de 2008). North Atlantic Council holds the first session of the nato-Georgia Commission. Recuperado de http://www.nato.int/ docu/update/2008/09-september/ e0915c.html

Organización del Tratado del Atlántico Norte (OTAN). (2 diciembre de 2015). Statement by NATO Foreign Minister on Open Door Policy. Recuperado de http://www.nato.int/cps/ en/natohq/official_texts_125591. $\mathrm{htm}$ ?selectedLocale $=$ en

Organización del Tratado del Atlántico Norte (OtAN). (julio, 2016). NATO Enlargement \& Open Door. Recuperado de https://www.nato. int/nato_static_fl2014/assets/pdf/ pdf_2016_07/20160627_1607factsheet-enlargement-eng. $\overline{p d f}$

Organización del Tratado del Atlántico Norte (OTAN). (15 de febrero de 2016). Montenegro begins Accession Talks with NATO. Recuperado de http://www.nato.int/ cps/en/natohq/news_128096. $\mathrm{htm}$ ?selectedLocale $=\mathrm{en}$

Organización del Tratado del Atlántico Norte (OtAN). (9 de julio de 2016). Warsaw Summit Communiqué. Recuperado de http://www. nato.int/cps/en/natohq/official_ texts_133169.htm\#open-door

Organización del Tratado del Atlántico Norte (OTAN). (24 de noviembre de 2016). Relations with Serbia. Recuperado de http://www.nato.int/cps/ en/natolive/topics_50100.htm
Organización del Tratado del Atlántico Norte (OtAN). (7 de junio de 2017). Partnership for Peace programme. Recuperado de http://www.nato. int/cps/en/natolive/topics_50349. htm

Organización del Tratado del Atlántico Norte (otAN). (9 de junio de 2017). Euro-Atlantic Partnership Council. Recuperado de http://www.nato. int/cps/en/natohq/topics_49276. htm

Organización del Tratado del Atlántico Norte (otan). (12 de junio de 2017). Membership Action Plan (MAP). Recuperado de http://www.nato.int/ cps/en/natolive/topics_37356.htm

Organización del Tratado del Atlántico Norte (OtAN). (11 de julio de 2017). Relations with Ukraine. Recuperado de http://www.nato.int/cps/en/ natolive/topics_37750.htm

Organización del Tratado del Atlántico Norte (otan). (23 de agosto de 2017). Relations with Georgia. Recuperado de http://www.nato.int/ cps/en/natolive/topics_38988.htm

Organización del Tratado del Atlántico Norte (OtAN). (2005). La seguridad en asociación. Bruselas: Organización del Tratado del Atlántico Norte (OTAN).

OTAN abre cumbre de Bucarest bajo presión de Bush por Georgia y Ucrania. (1 abril de 2008). La Nación. Recuperado de https:// www.nacion.com/el-mundo/ otanabre-cumbre-de-bucarest-bajopresion-de-bush-por-georgia-y- 
ucrania/2HOVXJTTD5BSXJMZ7T6ZOPUYRI/story/

OTAN- Putin pregunta "contra quién existe la OTAN" ahora que ya no está la uRSS y no hay riesgo de una nueva Guerra Fría. (4 de abril de 2008). Europa Press. Recuperado de http:// www.europapress.es/internacional/ noticia-otan-putin-pregunta-contra-quien-existe-otan-ahora-ya-nourss-no-hay-riesgo-nueva-guerrafria-20080404170238.html

Parere cGl sul Kosovo: processo farsa. (23 de julio de 2010). Osservatorio Italiano. Recuperado de http://osservatorioitaliano.org/read. php?id $=60861$

Priego, A. (2 de abril de 2014). Crimea revitaliza la OtAN. Real Instituto Elcano. Recuperado de https://blog. realinstitutoelcano.org/crimea-revitaliza-la-otan/

Requena, P. (5 de marzo de 2014). Ucrania, en el abismo. Instituto Español de Estudios Estratégicos, (23). Recuperado de http://www. ieee.es/Galerias/fichero/docs_opinion/2014/DIEEEO23-2014_Ucrania_en_el_Abismo_P.Requena.pdf

Rizzi, A. (17 de marzo de 2014). Crimea no es (exactamente) Kosovo. El País. Recuperado de https://elpais. com/internacional/2014/03/17/ actualidad/1395082939_669477. html
Rubio Plo, A. (3 de abril de 2014). Ucrania y la OTAN: una respuesta atenuada. Real instituto Elcano. Recuperado de https://blog. realinstitutoelcano.org/ucrania-yla-otan-una-respuesta-atenuada/

Ruiz, F. (6 marzo de 2014). Una visión alternativa de la crisis de Ucrania. Esglobal. Recuperado de https:// www.esglobal.org/una-vision-alternativa-de-la-crisis-de-ucrania/

Rynning, S. (2005). nato renewed. The Power and Purpose of Transatlantic Cooperation. Nueva York: Palgrave.

Smith, M. (2000). Nato in the First Decade after the Cold War. Dordrecht: Kluwer Academy Publishers.

Tribunal Penal Internacional para la ex Yugoslavia (TPIY). (2016). Key Figures of the Cases. Recuperado de http:// www.icty.org/en/cases/key-figurescases

Walker, E. (13 de abril de 2015). Between East \& West: Nato Enlargement \& the Geopolitics of the Ukraine Crisis. E-International Relations. Recuperado de http://www.e-ir.info/2015/04/13/ between-east-west-nato-enlargement-the-geopolitics-of-the-ukraine-crisis/

Wolff, A. (16 de septiembre de 2015). The future of NATO enlargement after the Ukraine crisis. International Affairs, 91(5), 1103-1121. 
